\title{
Anti-gitanismo, conocimiento racial y amnesia colonial
}

\author{
Anti-Gypsyism, Racial Knowledge and Colonial Amnesia
}

Giovanni Picker

University of Glasgow

Traducción de Ezequiel Martínez Llorente

\section{RESUMEN}

Este texto aúna visiones de las teorías críticas sobre la raza y de la sociología histórica para ofrecer un marco que permita comprender el extendido racismo contra el pueblo romaní a través de Europa. El texto se apoya directamente en la monografía Racial Cities (Picker, 2017). Argumenta que, para comprender la segregación racial de los romaníes en Europa, el conocimiento racial y la amnesia colonial han de resituarse en el centro del escrutinio analítico y de la intervención política. La razón para ello es que al observar cómo actúan diferentes autoridades urbanas sobre los romaníes en la Europa del siglo XXI, pueden identificarse similitudes clave entres esas acciones y las que ejercieron las autoridades coloniales sobre los "nativos" en las ciudades de los imperios europeos.

PALABRAS CLAVE: segregación racial, antigitanismo y colonialismo

\section{ABSTRACT}

This intervention brings together insights from race critical theories and historical sociology to provide a framework for understanding the longstanding racism against Romani people across Europe. It directly draws on Picker's 2017 monograph Racial Cities, and argues that in order to understand the racial segregation of Romani people in Europe, racial knowledge and colonial amnesia should be squarely placed at the core of analytical scrutiny and political 
intervention. The reason for this is that when looking at several cases of urban authorities' actions on Romani people in 21st-century Europe, key similarities can be detected with colonial authories's actions on "natives" in the cities of European empires.

KEY WORDS: racial segregation, antigypsyism, colonialism

\section{CONOCIMIENTO RACIAL}

«Pescara [zona centro de Italia], miércoles, 2 de mayo de 2012. Un joven de 24 años ha sido asesinado en la plaza de los Grue, durante una misión de castigo llevada a cabo, muy probablemente, por un grupo de nómadas». La página web de noticias CityRumors describía el escenario de los hechos, citando una larga historia de rivalidad entre los hinchas de fútbol locales y los «nómadas». En el artículo, al máximo sospechoso del crimen se le calificaba de «rom» (por «romaní»). Al día siguiente, el titular principal de la web era: «Pescara: Cócteles molotov, piedras, pancartas y manifestaciones contra los romaníes». Y se explicaba que: «Se respira una atmósfera de reyerta tras el asesinato de un hincha. A causa de la muerte, se ha extendido un clima contrario a los gitanos («zíngaros»), que ahora se extiende a todos los apellidos conocidos». A los dos días del crimen, un grupo de alrededor de 2.000 ciudadanos de Pescara se congregó delante del ayuntamiento. En la pancarta que portaban, podía leerse: «Tenéis cinco días para echarlos de la ciudad». Dos semanas más tarde, el alcalde de Pescara, miembro del partido de centro-derecha PDL [Popolo della Libertà] mandaba colocar carteles por toda la ciudad con el siguiente anuncio: «El PDL cumple sus promesas: jromaníes y criminales fuera de las viviendas sociales!».

Esta breve historia ilustra algunos de los elementos clave del anti-gitanismo en Europa, entre los que se cuentan la sospecha (quienes cometieron el asesinato «muy probablemente» fueron «un grupo de nómadas»), esa figura recurrente (tropo) del «nomadismo», la vergüenza pública, la escalada violenta, los sentimientos de vendetta y la discriminación legal (una sentencia judicial posteriormente condenaría al político del PDL por esas acciones). No obstante, en la crónica hay una expresión que tal vez se haya pasado por alto: «un clima contrario a los gitanos», puede leerse en la información, que «se extiende a todos los apellidos conocidos». ¿A qué quiere referirse el periodista con estos «apellidos conocidos»? 
Pescara fue una de las cinco ciudades donde, en varios periodos repartidos entre los años 2006 y 2015, llevé a cabo un extenso trabajo de campo sociológico con destino a mi libro Racial Cities: Governance and the Segregation of Romani People in Urban Europe [Routledge 2017]. Una vez inmerso en el contexto local, descubrí que una de las maneras que tienen los pescaresi no-romaníes para descubrir si alguien es gitano o no es precisamente a través de los apellidos. Tanto es así que puede afirmarse que hay unos «apellidos conocidos» que son inconfundiblemente «romaníes». Y a esto es a lo que se refería el artículo.

¿Qué son esos apellidos? Son indicadores de la ascendencia de cada uno, porque remiten a los antepasados y a la herencia personal. Así pues, al usar los apellidos como instrumento para identificar a una población sospechosa, como fue el caso en el asesinato de 2012, lo que está haciéndose es conectar la ascendencia (étnica) con un comportamiento (desviado). Esta es la definición de conocimiento racial. La raza es precisamente esa disposición previa por la que se establece (se construye) un nexo directo entre la ascendencia y un acto delictivo, entre lo natural y lo cultural, entre lo que está biológicamente determinado y lo que se comparte en términos morales. Este tipo de definición no es hoy tema de debate en Europa. Se ha silenciado (Lentin 2008) o «está fuera de toda mención, y solo cabe afirmar tal cosa en referencia al antisemitismo del pasado» (Goldberg 2006, 339). La mayoría de estudios y trabajos de investigación europeos hablan del racismo como una cuestión vinculada con las opiniones, como algo que se deriva por completo de las percepciones e ideas personales de cada uno. El sentido más frecuente que se le da en Europa al racismo es aquel que se refiere en exclusiva a «la vida de la mente». Y no puede haber nada más equivocado que esta forma de entender el racismo, porque el nexo directo entre el discurso de la ascendencia y el discurso de la moralidad no existe únicamente en las cabezas de la gente. También se encuentra en las legislaciones, en los currícula escolares y en las regulaciones económicas.

En Racial Cities, pude demostrar que el racismo en Europa existe también en las estructuras urbanas, en las decisiones que atañen a la política urbana (y en las indecisiones al respecto), y en la estructura y la organización de los espacios, cuando se pone el foco en determinadas áreas urbanas. Por toda Europa, existen áreas urbanas económicamente deprimidas, con pocos o nulos servicios públicos, a las que las autoridades locales generalmente ignoran o niegan, y que terminan siendo estigmatizadas como «zonas gitanas». Espacios así pueden hallarse en numerosas ciudades, como Bratislava (Lunik 9), Roma (Casilino 900), Madrid (Cañada Real) o el área de Mánchester (el campamento de Duchy Road). Estas presencias señalan un fenómeno urbano europeo que yo denomino «zonas 
urbanas gitanas». En Racial Cities, argumento que las condiciones que han propiciado la existencia y la perduración de estos espacios se basan en la raza como una manera precisamente de conectar el discurso de la biología con el de la conducta—, algo que históricamente ha tenido articulaciones muy variadas en los diferentes lugares.

En el libro, acuño un instrumento analítico, los «mecanismos de segregación local», para analizar las formas principales en que se establecen y se fijan las «zonas urbanas gitanas». Identifico los cuatro mecanismos más importantes en Europa, y les dedico sendos capítulos a su análisis: Desplazamiento (Cap. 2), Omisión (Cap. 3, el que trata de Pescara), Contención (Cap. 4) y Cohesión (Cap. 5). Cada uno de estos mecanismos sigue su propia lógica y posee unos referentes históricos singulares, y tal como los percibo, suponen declinaciones particulares de la ley racial. Esto significa que en todos puede apreciarse una connotación racial: por tanto, puede hablarse de «Desplazamiento racial», de «Contención racial», etc. Para entender la forma en que estos mecanismos son declinaciones locales de la ley racial, a continuación voy a tratar sobre el ejemplo etnográfico del desplazamiento, aquí a través del caso de una expulsión que tuvo lugar en Cluj-Napoca, en Rumanía, durante 2010.

En diciembre de 2010, 76 familias romaníes fueron expulsadas del centro de la ciudad y reubicadas en una zona aislada a 6 kilómetros de allí, sita a 300 metros del vertedero más grande en el norte de Rumanía. Paradójicamente, la justificación de toda esta operación eran las condiciones higiénicas de las viviendas de esas familias en el centro de la ciudad. Cuando entrevisté a las autoridades municipales que llevaron a cabo esa operación de desplazamiento, me topé con algunas representaciones peculiares de los roma, en las se hacía constante referencia a una supuesta «mentalidad» romaní (mentalitate en rumano). Esta mentalidad se asociaba a una dudosa disposición para el trabajo, junto a una cierta rigidez para conservar algunas supuestas tradiciones no compatibles con aquellas de la mayoría. Una de las extensiones de esto último era la falta de higiene con que se consideraba vivían los roma en el centro de la ciudad. Esa fue la razón oficial, alegada por el alcalde, para expulsar y reubicar a 76 familias.

Tras entrecruzar esta clase de datos empíricos con estudios sobre los significados y los valores de la mentalitate rumana, propuse una interpretación sobre cómo esas referencias a la higiene y la mentalidad están asociadas a una clase de razonamiento causante de operaciones como esta de desplazamiento, algo que se ampara en una representación racial de los roma. La raza, en este caso específico, 
está vinculada al hecho de que la mentalitate, ese tropo, se percibe como una condición inmodificable que supuestamente todos los roma poseen, y no tanto una característica cultural y por tanto susceptible de cambio. Desde esta perspectiva, la mentalitate consigue señalar a un grupo particular, los roma, como casi un cúmulo de representaciones hereditarias, con otras tantas connotaciones negativas, como la dudosa disposición al trabajo, y esa diferencia incompatible en cuanto a las tradiciones. Si bien no es estrictamente «biológico», el discurso de la mentalitate en referencia a los roma funcionó como un subtexto racial de la operación de desplazamiento. De este modo, cabe considerar el discurso de la mentalitate como conocimiento racial, ya que guarda similitud con las formas de conocimiento de «nativos» durante las colonias que surgieron con los primeros encuentros coloniales, como Goldberg (2002:23) explica:

La raza se impone sobre la otredad, en el intento de tomar nota de ella, de conocerla, de controlarla [...] Pero, paradójicamente, una vez configurada racialmente $[\ldots]$ esa amenaza comienza a magnificarse especialmente cargada, porque al ser nombrada racialmente, la amenaza se cosifica y se vuelve real, se realiza.

El desplazamiento puede por tanto considerarse un desplazamiento racial, vinculado a otros contextos de toda la Europa occidental. Las expulsiones de familias romaníes de alojamientos improvisados se han producido de forma constante en París, Madrid, Milán, Roma, Estocolmo y otras ciudades, y suponen una maniobra política recurrente, que los consistorios llevan adoptando desde comienzos del siglo XXI (Barker 2017; Fassin et al. 2014; Aguilera 2018; Vitale 2008). Al considerar la constitución racial del desplazamiento, no pretendo afirmar que cualquier estudio que aborde la marginalización de los roma y que no incluya la raza está equivocado. En lugar de eso, mi intención es señalar la presencia de una dimensión, la raza, que a menudo se pasa por alto en Europa, y no solo en los estudios de los fenómenos que afectan a los roma, sino en otras muchas áreas.

Para sustanciar mejor mi punto de vista, en el que defiendo que la raza juega un rol sustancial en el establecimiento y la regulación de «zonas urbanas gitanas», aporto más pruebas en el libro. Allí hago una recensión de la ideología y la lógica segregacionistas de tres ciudades colonizadas: la francesa Rabat, la británica Nueva Delhi y la italiana Addis Abeba. Con ello, trazo una línea socio-histórica de correspondencias entre los cuatro mecanismos segregacionistas en la Europa del siglo XXI y esas tres ciudades colonizadas. Así pues, por ejemplo, el 
desplazamiento racial, como un mecanismo local segregacionista, aplicado en Cluj-Napoca, por mucho que aluda al asunto de la higiene como justificación última de la operación de desplazamiento, parece guardar muchas similitudes con el razonamiento que llevó a segregar a los «nativos» en la Nueva Delhi británica. Edwin Lutyens, el arquitecto que firmó el plan de Nueva Delhi en 1924, tenía el objetivo de constreñir a los «nativos» dentro de una zona cercana al río Yamuna, separada por el Cordon Sanitaire de la «ciudad nueva», diseñada para los colonizadores blancos. Si bien la explotación laboral y, de forma más general, el beneficio económico de los británicos fueron motivaciones muy importantes para la segregación de los «nativos», la higiene fundamentó notoriamente el proyecto de Lutyens. Una gran cantidad de evidencias muestran que los miedos asociados a la propagación de enfermedades, también debido a las precarias infraestructuras de alcantarillado, desencadenaron uno de los debates más acalorados en la ciudad en ese momento. Y la lógica organizativa de la dominación colonial, ciertamente, se basaba por encima de todo en la raza.

El resultado de este intento integrado para mostrar las similitudes entre los mecanismos segregacionistas locales que afectan a los romaníes hoy en Europa y la lógica de la segregación imperante en las ciudades colonizadas europeas es el objeto de un cuadro analítico que examino por extenso en el Capítulo 6 de Racial Cities (Figura 1).

\begin{tabular}{|c|c|c|c|}
\hline Colonized city & $\begin{array}{l}\text { Major colonial } \\
\text { ideology }\end{array}$ & $\begin{array}{l}\text { Major segregation } \\
\text { rationale }\end{array}$ & GUAs \\
\hline $\begin{array}{l}\text { French } \\
\text { Rabat }\end{array}$ & $\begin{array}{l}\text { Protection of local } \\
\text { culture }\end{array}$ & $\begin{array}{l}\text { Cultural } \\
\text { preservation }\end{array}$ & $\begin{array}{l}\text { Compi nomadi } \\
\text { (Florence) }\end{array}$ \\
\hline $\begin{array}{l}\text { British } \\
\text { New Delhi }\end{array}$ & $\begin{array}{l}\text { Wealth: commerce } \\
\text { and industry; } \\
\text { Overseas hegemony }\end{array}$ & $\begin{array}{l}\text { Hygiene and } \\
\text { sanitary concerns }\end{array}$ & $\begin{array}{l}\text { Colonia noua } \\
\text { (Cluj-Napoca); } \\
\text { "campsite" (Salford) }\end{array}$ \\
\hline $\begin{array}{l}\text { Italian } \\
\text { Addis Ababa }\end{array}$ & $\begin{array}{l}\text { Demographic } \\
\text { colonialism; } \\
\text { Re-imposing white } \\
\text { supremacy in East } \\
\text { Africa }\end{array}$ & $\begin{array}{l}\text { Enforcing social } \\
\text { order }\end{array}$ & $\begin{array}{l}\text { Rancitelli (Pescara); } \\
\text { Campi nomadi } \\
\text { (Florence); } \\
\text { "campsite" (Salford) }\end{array}$ \\
\hline
\end{tabular}

Figura 1. Ideologías coloniales, lógicas de segregación y «zonas urbanas gitanas» segregadas y estigmatizadas (Picker 2017: 133) 


\section{AMNESIA COLONIAL}

Una de las razones por las que decidí incluir un análisis de la segregación en las ciudades colonizadas, además de para subrayar la relevancia de la raza en la Europa del siglo XXI, fue para señalar la notable ausencia del colonialismo en los debates en los medios de comunicación, la escuela y los currícula universitarios, así como en el discurso político, en toda Europa. Un ejemplo muy revelador de esto último es el cargo creado por Ursula von der Leyen, presidenta electa de la Comisión Europea en 2019, denominado «vicepresidencia para la protección de nuestro modo de vida europeo». En un tweet aparecido el 12 de septiembre de $2019^{*}$, la presidenta electa explicaba que "nuestro modo de vida europeo» se basa en el artículo 2 del Tratado de Lisboa, el cual se abre con la siguiente afirmación: «La Unión [Europea] se funda en los valores del respeto a la dignidad humana, la libertad, la democracia, la igualdad, el Estado de derecho y el respeto a los derechos humanos». Como Hansen y Jonsson (2017) han detallado por extenso: «La gran correlación entre la integración europea y el colonialismo europeo, un hilo constatable para todo el que se moleste en buscar en los documentos de los archivos, ha recibido comparativamente poca atención en los estudios académicos recientes y contemporáneos». Los autores prosiguen para explicar que el proyecto de Euráfrica, nacido de las cenizas de la Segunda Guerra Mundial, estuvo presente en la constitución de la idea de una Europa unificada, dándose por supuesta la extensión de la posición subalterna de África tras siglos de reparto y dominación del territorio africano. Sin embargo, raramente se alude siquiera a este proyecto neo-colonial.

La amnesia colonial también afecta a los análisis y las teorías que puedan realizarse sobre las ciudades europeas. Un campo de trabajo académico que Racial Cities analiza críticamente son los estudios urbanos dedicados a la segregación en ciudades europeas. Ninguno de los análisis en torno a este fenómeno, rigurosos en cuanto al método y a la deducción teórica, hace mención alguna a la raza (ej. Weir 1993; van Kempen y Ozüekren 1998; Musterd 2005). Ciertamente, la raza puede que no juegue un papel clave en determinados contextos, pero es digno de notarse que estos estudios basan sus hipótesis en datos estadísticos consultables, como los censos nacionales, los cuales -en Europa, salvo en el Reino Unido- no incluyen ningún indicador que mencione explícitamente ninguna categorización racial. Esto equivale casi a sugerir que la raza solo existe si hay datos estadísticos que la mencionen explícitamente. Esta paradoja parte en gran medida de una de las verdades convencionales más consolidadas en los estudios sobre la segregación en Europa: que, para entender mejor a la Europa occidental, es útil la piedra de toque de los Estados Unidos. Y, 
una vez efectuada la comparación, podría concluirse así que la raza es un fenómeno estructural solo presente en los Estados Unidos, ya que la presencia de categorías raciales en su censo permite al investigador mapear ciudades de acuerdo con las adscripciones raciales de los hogares y la gente.

Frente a esto, lo que sugiero en Racial Cities es que, en lugar de comparar Europa con Estados Unidos, los estudios sobre las ciudades europeas deberían investigar históricamente el urbanismo colonial, en su condición de uno de los primeros experimentos urbanos que obedecieron a teorías planificadoras europeas impuestas; verdaderamente, los primeros experimentos urbanos en abordar la heterogeneidad social y la «diferencia humana» han de resultar claves para entender la génesis de un asunto tan de esta época: las relaciones entre la planificación urbanística y la «diversidad urbana» en Europa. Al dirigir la vista al urbanismo colonial, sugiero, uno puede entender las continuidades (y discontinuidades) entre las concepciones coloniales y contemporáneas y sus prácticas derivadas sobre planificación, coexistencia, diversidad e igualdad.

Apoyándome en prominentes expertos postcoloniales como Césaire (1950) y Fanon (2004 [1963]), entre otros, una de las conclusiones fundamentales de Racial Cities es que la investigación del anti-gitanismo, que es quintaesencialmente una forma europea de racismo, podría salir beneficiada si dedicara una mayor atención a las formas de conocimiento racial y de gobernanza que fueron moneda común en el trato directo con los «nativos» en los contextos colonizados. Así pues, por ejemplo, la extendida práctica de segregar a personas romaníes en campamentos, tan común en Italia y el Reino Unido, pero tampoco rara en Francia, es más comprensible si se contextualizan las varias formas de conocimiento y gobernanza raciales que afectaron a los nativos en el mundo colonizado. En su sociología histórica sobre la gobernación de poblaciones consideradas «diferentes», Magubane (2004) explica cómo en el Reino Unido la figura recurrente del «nomadismo», una forma muy común de estigmatizar a la población romaní en toda Europa, está vinculada en su origen con las representaciones de los sirvientes khoikhoi y las personas esclavizadas a las que se criminalizó con la Cape Vagrancy Ordinance de 1834. Este hecho, por ejemplo, podría aportar una información de gran interés sobre el modo en que el tropo cultural (racista) del nomadismo ha arraigado en Europa, y hasta qué punto acarrea formas de conocimiento racial, algo con una presencia tan ubicua y poderosa en las colonias. 


\section{CONCLUSIÓN}

Esto me conduce a esta breve nota conclusiva sobre la importancia de analizar la historia del anti-gitanismo, labor de la que se ocupa este número especial de Sociología Histórica. La situación de pobreza y exclusión social que el $80 \%$ de los hogares romaníes europeos encaran (EU-MIDIS II, 2016) es demasiado seria como para no darle un enfoque histórico a la cuestión. El modo en que formas sedimentadas de conocimiento y amnesias actuales pueden contribuir para impedir el cambio social debería tener una alta prioridad en las agendas académicas. Los legisladores también podrían beneficiarse si contaran con una perspectiva histórica acerca de políticas, leyes y medidas institucionales pasadas, a la hora de tratar directamente con una minoría europea que ha sido mantenida en gran medida en situaciones de desposesión e injusticia a lo largo de toda la historia europea. Una historia que, como muestra Racial Cities, ha de ser considerada en todas sus dimensiones, entre las que se cuenta el colonialismo.

\section{REFERENCIAS}

AGUILERA, T. 2018. «Reloger, mettre en attente et expulser les bidonvilles de Madrid: quand des politiques de résorption produisent de l'expulsion», L'Année sociologique, vol. 68(1), 101-134.

BARKER, V. 2017. Nordic vagabonds: The Roma and the logic of benevolent violence in the Swedish welfare state, European Journal of Criminology, 14(1), 120-139.

CÉSAIRE, Aimé. 1955. Discours sur le colonialisme, París, Editions Présence Africaine. [Discurso sobre el colonialismo, Madrid, 2015]

EU-MIDIS II, 2016. Second European Union Minorities and Discrimination Survey, Viena, Fundamental Rights Agency.

FANON, Frantz. 2004[1963]. The Wretched of the Earth, Nueva York, Grove Press. [Los condenados de la tierra, Navarra, Txalaparta, 1999]

FASSIN, Eric, Carine Fouteau, Serve Guichard y Aurélie Windels. 2014. Roms \& Riverains. Une politique municipal de la race, París, La Fabrique.

GOLDBERG, D.T. 2006. «Racial Europeanization», Ethnic and Racial Studies.

GOLDBERG, D.T. 2002. The Racial State. Oxford, Blackwell. 
HANSEN, P. \& Jonsson, S. 2017. «Eurafrica Incognita: The Colonial Origins of the European Union", History of the Present, 7(1), 1-32.

LENTIN, A. 2008. «Europe and the Silence About Race», European Journal of Social Theory, 11 (4), 487-503.

MAGUBANE, Zine. 2004. Bringing The Empire Home: Race, Class and Gender in Britain and Colonial South Africa, Chicago, University of Chicago Press.

PICKER, G. 2017. Racial Cities: Governance and the Segregation of Romani People in Urban Europe, Abingdon y Nueva York, Routledge.

VAN KEMPEN, Ronald y A. Ozüekren. 1998. «Ethnic Segregation in Cities: New Forms and Explanations in a Dynamic World», CURS 35(10), 1631-1656.

VITALE, T. 2008. «Etnografia degli sgomberi di un insediamento rom a Milano. L'ipotesi di una politica locale eugenetica», Mondi migranti, 11 (1), 59-74.

WEIR, Margaret. 1993. «Race and Urban Poverty: Comparing Europe and America», Brookings Review, 1 (1), 22-27.

Recibido: 10 de septiembre de 2019

Aceptado: 15 de octubre de 2019

Gionanni Picker es Profesor de Sociología en la Univesidad de Glasgow. Es autor del libro Racial Cities: Governance and the Segregation of Romani People in Urban Europe (Routledge 2017) y coeditor del volume Racialized Labour in Romania: Spaces of Marginality at the Periphery of Global Capitalism (Palgrave 2018). Ha publicado sus trabajos, entre otras, en las siguientes revistas: International Journal of Urban and Regional Research, Social Identities, CITY, International Sociology, Identities and Journal of Contemporary Ethnography. Giovanni is also the coordinator for Central and Eastern Europe and Russia of the Amsterdam-based, independent Summer School on Black Europe: Interrogating Citizenship, Race and Ethnic Relations. 